Filomat 31:20 (2017), 6551-6560

https://doi.org/10.2298/FIL1720551B

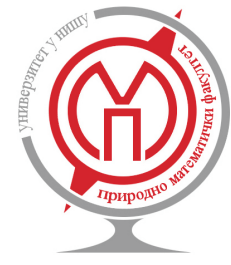

Published by Faculty of Sciences and Mathematics, University of Niš, Serbia

Available at: http://www.pmf.ni.ac.rs/filomat

\title{
The Diameter of Cyclic Kautz Digraphs
}

\author{
Katerina Böhmováa ${ }^{\text {, Cristina Dalfób }}{ }^{\text {, Clemens Huemer }}{ }^{\mathrm{b}}$ \\ ${ }^{a}$ Dept. of Computer Science, ETH Zürich, Switzerland \\ ${ }^{b}$ Dept. de Matemàtica, Universitat Politècnica de Catalunya, Barcelona, Catalonia
}

\begin{abstract}
We present a new kind of digraphs, called cyclic Kautz digraphs $C K(d, \ell)$, which are subdigraphs of the well-known Kautz digraphs $K(d, \ell)$. The latter have the smallest diameter among all digraphs with their number of vertices and degree.

Cyclic Kautz digraphs $C K(d, \ell)$ have vertices labeled by all possible sequences $a_{1} \ldots a_{\ell}$ of length $\ell$, such that each character $a_{i}$ is chosen from an alphabet containing $d+1$ distinct symbols, where the consecutive characters in the sequence are different (as in Kautz digraphs), and now also requiring that $a_{1} \neq a_{\ell}$. Their arcs are between vertices $a_{1} a_{2} \ldots a_{\ell}$ and $a_{2} \ldots a_{\ell} a_{\ell+1}$, with $a_{1} \neq a_{\ell}$ and $a_{2} \neq a_{\ell+1}$.

We give the diameter of $C K(d, \ell)$ for all the values of $d$ and $\ell$, and also its number of vertices and arcs.
\end{abstract}

\section{Introduction}

It is well-known that, for integers $d \geq 2$ and $\ell \geq 1$, Kautz digraphs $K(d, \ell)$ have vertices labeled by all possible sequences $a_{1} \ldots a_{\ell}$ of length $\ell$ with different consecutive symbols, $a_{i} \neq a_{i+1}$ for $i=1, \ldots, \ell-1$, from an alphabet $\Sigma$ of $d+1$ distinct symbols. Kautz digraphs $K(d, \ell)$ have arcs between vertices $a_{1} a_{2} \ldots a_{\ell}$ and $a_{2} \ldots a_{\ell} a_{\ell+1}$. See Figure 1 .

In this paper we define cyclic Kautz digraphs $C K(d, \ell)$ (see Figure 2), where the labels of their vertices are defined as the ones of Kautz digraphs, with the additional requirement that the first and last symbols must also be different $\left(a_{1} \neq a_{\ell}\right)$. Cyclic Kautz digraphs $C K(d, \ell)$ have arcs between vertices $a_{1} a_{2} \ldots a_{\ell}$ and $a_{2} \ldots a_{\ell} a_{\ell+1}$, with $a_{i} \neq a_{i+1}, a_{1} \neq a_{\ell}$ and $a_{2} \neq a_{\ell+1}$. We observe that cyclic Kautz digraphs $C K(d, \ell)$ are subdigraphs of Kautz digraphs $K(d, \ell)$. Unlike in $K(d, \ell)$, any label of a vertex of $C K(d, \ell)$ can be cyclically

2010 Mathematics Subject Classification. 05C20

Keywords. Digraph, Diameter, Kautz digraph

Received: 13 June 2016; Revised: 14 March 2017; Accepted: 05 September 2017

Communicated by Francesco Belardo

K. Böhmová is a recipient of the Google Europe Fellowship in Optimization Algorithms, and this research is supported in part by this Google Fellowship. Research of C. Dalfó is supported by projects MINECO MTM2014-60127-P, and AGAUR 2014 SGR1147. Research of C. Huemer is supported by projects MINECO MTM2015-63791-R, and AGAUR 2014SGR46. C. Dalfó and C. Huemer have also received funding from the European Union's Horizon 2020 research and innovation programme under the Marie SkłodowskaCurie grant agreement No 734922.

Email addresses: katerina.boehmova@inf.ethz.ch (Katerina Böhmová), cristina.dalfo@upc.edu (Cristina Dalfó), clemens.huemer@upc.edu (Clemens Huemer)

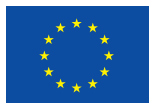

C. Dalfó and C. Huemer have also received funding from the European Union's Horizon 2020 research and innovation programme under the Marie Skłodowska-Curie grant agreement No 734922. 

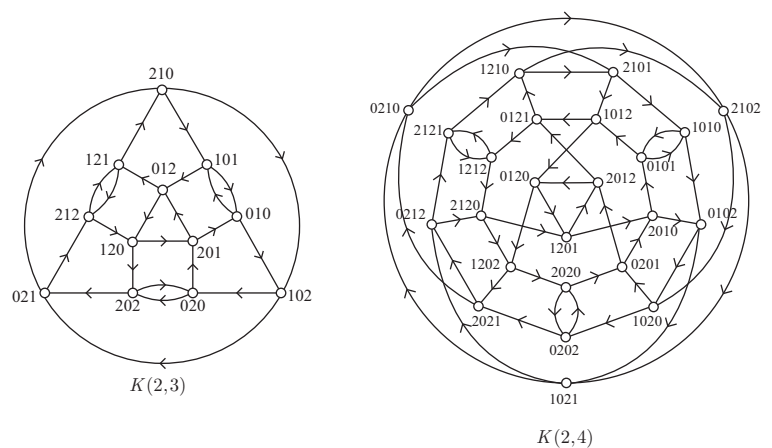

Figure 1: Kautz digraphs $K(2,3)$ and $K(2,4)$.

shifted to form again a label of a vertex of $C K(d, \ell)$. We study some of the properties of cyclic Kautz digraphs. Note that, in contrast to Kautz digraphs, $C K(d, \ell)$ are not $d$-regular (neither $d$-out-regular). Therefore, in $C K(d, \ell), d$ is the size of the alphabet minus one. Besides, if $\ell>3$ and $d>1$, the maximum out-degree of $C K(d, \ell)$ is $d$.

Cyclic Kautz digraphs $C K(d, \ell)$ could be relevant in coding theory, because they are related to cyclic codes. A linear code $C$ of length $\ell$ is called cyclic if, for every codeword $c=\left(c_{1}, \ldots, c_{\ell}\right)$, the codeword $\left(c_{\ell}, c_{1}, \ldots, c_{\ell-1}\right)$ is also in $C$. This cyclic permutation allows to identify codewords with polynomials. For more information about cyclic codes and coding theory, see Van Lint [8] (Chapter 6). With respect to other properties of cyclic Kautz digraphs $C K(d, \ell)$, their number of vertices follows sequences that have several interpretations. For example, for $d=2$ (that is, 3 different symbols), the number of vertices follows the sequence 6, 6, 18,30,66,... According to the On-Line Encyclopedia of Integer Sequences [9], this is the sequence A092297. For $d=3$ (4 different symbols) and $\ell=2,3, \ldots$, we get the sequence $12,24,84,240,732, \ldots$ corresponding to A226493 and A218034 in [9].

Originally, Kautz digraphs were introduced by Kautz [6, 7] in 1968. They have many applications, for example, they are useful as network topologies for connecting processors. Kautz digraphs $K(d, \ell)$ have order $d^{\ell}+d^{\ell-1}$, where $d$ is the degree and also is equal to the cardinality of the alphabet minus one. They are $d$-regular, have diameter $D=\ell$, and maximum connectivity (see Fàbrega and Fiol [4]). In fact, Kautz digraphs have the smallest diameter among all digraphs with their number of vertices and degree. Kautz digraphs $K(d, \ell)$ are related to De Bruijn digraphs $B(d, \ell)$, which are defined in the same way as Kautz digraphs, but without the restriction that adjacent symbols in the label of a vertex have to be distinct. Thus, Kautz digraphs are induced subdigraphs of De Bruijn digraphs, which were introduced by De Bruijn [3] in 1946. De Bruijn digraphs have order $d^{\ell}$, where the degree $d$ is equal to the cardinality of the alphabet, and they are also $d$-regular, have diameter $D=\ell$, and high connectivity. Another interesting property of Kautz and De Bruijn digraphs is that they can be defined as iterated line digraphs of complete symmetric digraphs and complete symmetric digraphs with a loop on each vertex, respectively (see Fiol, Yebra and Alegre [5]). Note that Kautz and De Bruijn digraphs are often inaccurately referred to as Kautz and De Bruijn 'graphs', which should not be confused with the underlying (undirected) Kautz and De Bruijn graphs.

In Section 2 of this paper we give the main parameters of the newly defined cyclic Kautz digraphs $C K(d, \ell)$, that is, the number of vertices, number of arcs, and diameter.

We use the habitual notation for digraphs, that is, a digraph $G=(V, E)$ consists of a (finite) set $V=V(G)$ of vertices and a set $E=E(G)$ of arcs (directed edges) between vertices of $G$. There are no multiple arcs, that is, there is at most one arc from each vertex to any other. If $a=(u, v)$ is an arc between vertices $u$ and $v$, then vertex $u$ is adjacent to vertex $v$, and vertex $v$ is adjacent from $u$. Let $\Gamma_{G}^{+}(v)$ and $\Gamma_{G}^{-}(v)$ denote the set of vertices adjacent from and to vertex $v$, respectively. Their cardinalities are the out-degree $\delta_{G}^{+}(v)=\left|\Gamma_{G}^{+}(v)\right|$ of vertex $v$, and the in-degree $\delta_{G}^{-}(v)=\left|\Gamma_{G}^{-}(v)\right|$ of vertex $v$. For all $v \in V$, a digraph $G$ is called $d$-out-regular if $\delta_{G}^{+}(v)=d$, $d$-in-regular if $\delta_{G}^{-}(v)=d$, and $d$-regular if $\delta_{G}^{+}(v)=\delta_{G}^{-}(v)=d$.

Given two positive integers $d_{0}$ and $\ell_{0}$, we use $C K\left(d \geq d_{0}, \ell \geq \ell_{0}\right)$ instead of the longer notation $C K(d, \ell)$ 
for $d \geq d_{0}$ and $\ell \geq \ell_{0}$.

For a vertex $v=v_{1} v_{2} \ldots v_{\ell}$, let $v_{i}$ denote the $i$-th symbol of $v$.

\section{Parameters of cyclic Kautz digraphs}
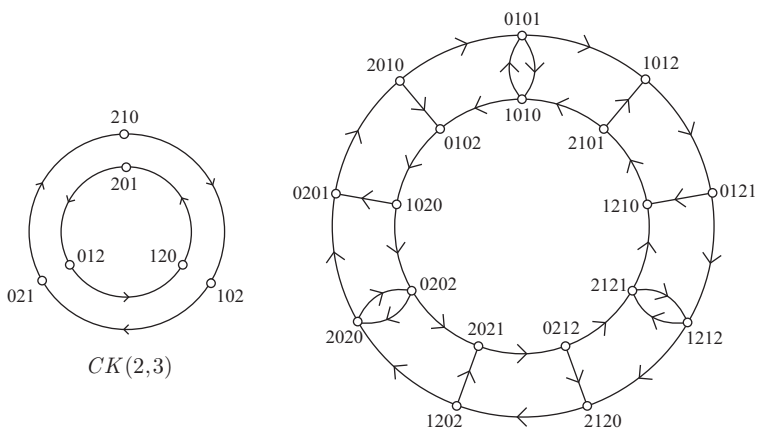

$C K(2,4)$

Figure 2: Cyclic Kautz digraphs $C K(2,3)$ and $C K(2,4)$.

In this section we provide the number of vertices, number of arcs, and diameter of cyclic Kautz digraphs $C K(d, \ell)$.

\subsection{Numbers of vertices and arcs}

The following two results yield the number of vertices and number of arcs of cyclic Kautz digraphs $C K(d, \ell)$.

Proposition 2.1. For $d \geq 2$ and $\ell \geq 2$, the number of vertices of cyclic Kautz digraph $C K(d, \ell)$ is

$$
n_{d, \ell}=(-1)^{\ell} d+d^{\ell} .
$$

Proof. There is a direct bijection between the vertices of $C K(d, \ell)$ and the closed walks of length $\ell$ in the complete symmetric digraph with $d+1$ vertices (which is equivalent to the complete graph): A vertex of $C K(d, \ell)$ is a sequence $a_{1} a_{2} \ldots a_{\ell}$ of different consecutive symbols, and with $a_{1} \neq a_{\ell}$, from an alphabet of $d+1$ distinct symbols. Such a sequence corresponds to a closed walk $a_{1} a_{2} \ldots a_{\ell} a_{1}$ in the complete graph with $d+1$ vertices. The claim now follows from the fact that the number of closed walks of length $\ell$ in a graph equals the trace of $A^{\ell}$, where $A$ is the adjacency matrix of the graph (see, for example, Brouwer and Haemers [2]). The spectrum of a complete graph with $d+1$ vertices has eigenvalue -1 with multiplicity $d$, and eigenvalue $d$ with multiplicity 1 . Therefore, for this graph, the trace of $A^{\ell}$ is $(-1)^{\ell} d+d^{\ell}$.

Instead of using spectral theory, a combinatorial proof of Proposition 2.1 is also possible, but it would be a bit longer.

Proposition 2.2. For $d \geq 2$ and $\ell \geq 2$, the number of arcs of cyclic Kautz digraph $C K(d, \ell)$ is

$$
m_{d, \ell}=(d+1) d^{\ell}-(2 d-1)\left((-1)^{\ell-1} d+d^{\ell-1}\right) .
$$

Proof. Note that for $\ell=2, C K(d, 2)$ is Kautz digraph $K(d, 2)$ and its number of arcs is $(d+1) d^{2}$. It is easily verified that for $\ell=3, C K(d, \ell)$ has $(d-1)^{2} d(d+1)$ arcs. Thus let $\ell>3$.

A vertex $v=a_{1} a_{2} \ldots a_{\ell}$ of $C K(d, \ell)$ is adjacent to vertices of the form $a_{2} \ldots a_{\ell} a_{\ell+1}$, where $a_{\ell+1} \neq a_{2}, a_{\ell}$. We distinguish two cases:

(a) If $a_{\ell}=a_{2}$, then vertex $v$ is adjacent to $d$ vertices that have the form $a_{2} \ldots a_{\ell-1} a_{2} a_{\ell+1}$, and their number is $d n_{d, \ell-2}$. This is because $a_{2} a_{3} \ldots a_{\ell-1}$ has length $\ell-2$ and corresponds to the number of vertices $n_{d, \ell-2}$. 
(b) If $a_{\ell} \neq a_{2}$, then vertex $v$ is adjacent to $d-1$ vertices having the form $a_{2} \ldots a_{\ell} a_{\ell+1}$ with $a_{\ell+1} \neq a_{2}$, and their number is $n_{d, \ell}-d n_{d, \ell-2}$.

Thus, by adding up the arcs described in $(a)$ and $(b)$, that is, $m_{d, \ell}=d^{2} n_{d, \ell-2}+\left(n_{d, \ell}-d n_{d, \ell-2}\right)(d-1)$, we obtain the result.

\subsection{Diameter}

In this section we compute the diameter of cyclic Kautz digraphs $C K(d, \ell)$, depending on the values of $d$ and $\ell$.

In the following claims and proofs, let us fix $\Sigma=\{0,1, \ldots, d\}$ to be the alphabet of $C K(d, \ell)$.

First, we discuss the diameter for small values of $d$ and $\ell$. Then, we deal with the general case $d \geq 4$ and $\ell \geq 4$.

Lemma 2.3. The diameter of $C K(d, 1)$ is 1 . Cyclic Kautz digraphs $C K(1, \ell \geq 2)$ exist only if $\ell$ is even. For even $\ell$, the diameter of $C K(1, \ell \geq 2)$ is 1 . The diameter of $C K(d \geq 2,2)$ is 2 .

The proof of this lemma is straightforward and omitted.

Each vertex $v$ of cyclic Kautz digraph $C K(d, \ell)$ can be uniquely represented on a disc with a marked start (see Figure 3). We will refer to this as a disc representation of vertex $v$ (in short, the disc of $v$ ). In fact, there is a straightforward bijection between the vertices of $C K(d, \ell)$ and the set of discs $D(d, \ell)$ with a marked start, containing $\ell$ symbols of the alphabet of size $d+1$ in such a way that no two consecutive symbols are the same. Moreover, vertex $u$ is adjacent to vertex $v$ if and only if the disc of $v$ can be obtained from the disc of $u$ by swapping the marked symbol (that is, changing it to a symbol, perhaps itself, different from its left and right neighbors), and rotating the disc one position. This gives directly the proof of the following lemma, so it is omitted.
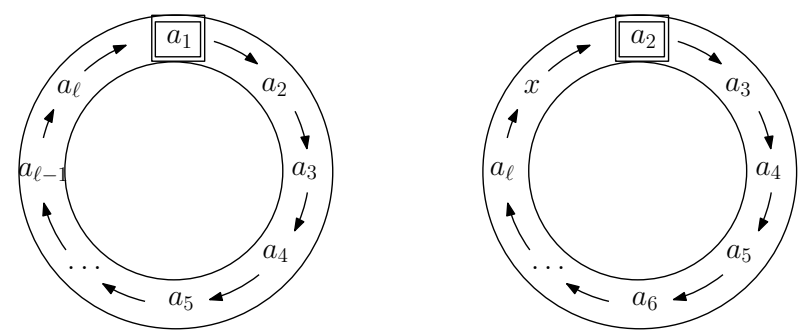

Figure 3: The disc (or circular) representations of a vertex $u=a_{1} a_{2} \ldots a_{\ell-1} a_{\ell}$ of $C K(d, \ell)$ and its neighbor $v=a_{2} a_{3} \ldots a_{\ell} x$, that is, $(u, v)$ is an $\operatorname{arc}$ in $C K(d, \ell)$.

Lemma 2.4. There is a path from a vertex $u$ to a vertex $v$ in $C K(d, \ell)$ if and only if the disc of $v$ can be obtained from the disc of $u$ by a sequence of operations:

- Rotation of the disc.

- Swap of one symbol.

Let us now consider cyclic Kautz digraphs $C K(2, \ell)$ with alphabet $\Sigma=\{0,1,2\}$, and let us define a function sgn : $\Sigma^{2} \rightarrow\{+,-\}$, which assigns a $+/-$ sign to an ordered pair of distinct symbols $(a, b)$ as follows:

$$
\begin{aligned}
& \operatorname{sgn}(0,1)=\operatorname{sgn}(1,2)=\operatorname{sgn}(2,0)=+, \\
& \operatorname{sgn}(1,0)=\operatorname{sgn}(2,1)=\operatorname{sgn}(0,2)=-.
\end{aligned}
$$

Given a vertex $v$ of $C K(2, \ell)$, for $\ell>1$ we define an imprint of $v$ as a sequence $i m(v)$ of length $\ell$ containing symbols + and - as follows. On the $i$-th position $(i \in\{1, \ldots, \ell-1\})$ of the sequence $i m(v)$ there is the symbol $\operatorname{sgn}\left(v_{i}, v_{i+1}\right)$. On the last position of $i m(v)$ there is the symbol $\operatorname{sgn}\left(v_{\ell}, v_{1}\right)$. Since each two consecutive symbols of $v$ are distinct, the imprint of $v$ is well defined and unique. 
Lemma 2.5. Let $u$ and $v$ be two vertices of $C K(2, \ell)$, for $\ell>1$. The imprints of $u$ and $v$ have the same number of + and - signs if and only if the disc of $v$ can be obtained from the disc of $u$ by a sequence of the operations in Lemma 2.4.

Proof. To prove the reverse implication, we should first observe that performing any of the two operations does not change the number of $+/-$ signs in the imprint. Rotation clearly has no influence on the number of + and - signs in the imprint. The swap of a symbol $u_{i}$ can only be performed if $u_{i-1}=u_{i+1}$.

To show the forward implication, let us now consider two vertices $u$ and $v$ of $C K(2, \ell)$ such that the numbers of + and - signs in the two imprints agree. We will show that this implies that the disc of $v$ can be obtained from the disc of $u$ by a sequence of the mentioned operations as follows. Since there are only 3 different symbols in $u$ and $v$, there is a symbol that appears in both of $u$ and $v$, say $u_{s}=v_{t}$. Using rotations, there is no loss of generality if we assume that $s=t=1$.

Note that rotating the disc of a vertex $u=u_{1} u_{2} \ldots u_{\ell}$ by one position yields the disc of a vertex $u^{(1)}=$ $u_{2} \ldots u_{\ell} u_{1}$, and $\left(u, u^{(1)}\right)$ is an arc of $C K(2, \ell)$. Then, by rotating the disc of $u$ by $k$ positions, we obtain a vertex $u^{(k)}=u_{k+1} \ldots u_{\ell} u_{1} \ldots u_{k}$ and there is a path from $u$ to $u^{(k)}$ in $C K(2, \ell)$. The same transformation can be applied to any other vertex, say, $v$ or $w$.

Now observe that by swapping the symbol at a position $i$ in the disc of a vertex $w$, we can change the imprint of $w$ in positions $i-1$ and $i$ from -+ to +- or vice versa. For example, if $\operatorname{sgn}\left(w_{i-1}, w_{i}\right)=-$ and $\operatorname{sgn}\left(w_{i}, w_{i+1}\right)=+$, then $w_{i-1}=w_{i+1}$, and thus, we can swap the symbol $w_{i}$ for the remaining symbol (different from $w_{i-1}$ and $w_{i}$ ). Based on this observation, notice that by a sequence of operations of swapping a symbol of the disc of $w=w_{1} \ldots w_{\ell}$, we can obtain the disc of $w^{\prime}=w_{1}^{\prime} \ldots w_{\ell}^{\prime}$ such that $w_{1}^{\prime}=w_{1}$ and the imprint im $\left(w^{\prime}\right)=(+\ldots+-\ldots-)$ contains all the + signs contiguously at the beginning and all the - signs contiguously at the end. Thus, in particular, we can transform by a sequence of swaps the discs of vertices $u^{\prime}=u_{1}^{\prime} \ldots u_{\ell}^{\prime}$ and $v^{\prime}=v_{1}^{\prime} \ldots v_{\ell}^{\prime}$ into the discs of vertices $u^{\prime \prime}=u_{1}^{\prime \prime} \ldots u_{\ell}^{\prime \prime}$ and $v^{\prime \prime}=v_{1}^{\prime \prime} \ldots v_{\ell}^{\prime \prime}$ such that $u_{1}^{\prime \prime}=u_{1}^{\prime}=v_{1}^{\prime}=v_{1}^{\prime \prime}$, and both imprints $i m\left(u^{\prime \prime}\right)$ and $i m\left(v^{\prime \prime}\right)$ are of the form $(+\ldots+-\ldots-)$. Since the imprints of $u$ and $v$ have the same number of $+/-$ signs and the performed operations do not change it, it follows that $i m\left(u^{\prime \prime}\right)=\operatorname{im}\left(v^{\prime \prime}\right)$.

Finally, observe that the imprint of a vertex $w$ together with the first symbol of $w$ determines uniquely the vertex $w$ in $C K(2, \ell)$. Since $i m\left(u^{\prime \prime}\right)=i m\left(v^{\prime \prime}\right)$ and $u_{1}^{\prime \prime}=v_{1}^{\prime \prime}$, we obtain that $u^{\prime \prime}=v^{\prime \prime}$. Therefore, the disc of $v$ can be obtained from the disc of $u$ by a sequence of the mentioned operations.

Then, from Lemmas 2.4 and 2.5, we have the following result.

Corollary 2.6. In $C K(2, \ell)$, there is a path from a vertex $u$ to a vertex $v$ if and only if the imprint of $v$ contains the same number of + and - signs as the imprint of $u$.

We now use Corollary 2.6 to prove the following result.

Table 1: Two vertices $u$ and $v$ of $C K(2, \ell)$, for $\ell=3$ and for $\ell \geq 5$, such that the numbers of + and - signs in their imprints differ, implying that $u$ and $v$ belong to different strongly connected components.

\begin{tabular}{l|c|c} 
& $u$, imprint of $u$ & $v$, imprint of $v$ \\
\hline$\ell=3$ & 012 & 021 \\
& $(+++)$ & $(---)$ \\
\hline$\ell=3 r, \ell \geq 5$ & $012012 \ldots 012$ & $021012 \ldots 012$ \\
& $(++++++\ldots+++)$ & $(---+++\ldots+++)$ \\
\hline$\ell=3 r+1, \ell \geq 5$ & $012012 \ldots 0121$ & $021012 \ldots 0121$ \\
& $(++++++\ldots++--)$ & $(---+++\ldots++--)$ \\
\hline$\ell=3 r+2, \ell \geq 5$ & $012012 \ldots 01201$ & $021012 \ldots 01201$ \\
& $(++++++\ldots++++-)$ & $(---+++\ldots++++-)$
\end{tabular}

Lemma 2.7. The diameter of $C K(2,4)$ is $7 . C K(2,3)$ and $C K(2, \ell \geq 5)$ are not strongly connected. 
Proof. As it can be checked in Figure 2, the diameter of $C K(2,4)$ is $D=7$. We could argue that the diameter of $C K(2,4)$ is finite by showing that all the vertices of $C K(2,4)$ have imprints that contains exactly two + signs and two - signs. Since the size of the alphabet is 3 , any vertex $v$ of $C K(2,4)$ contains some symbol twice and it cannot be on neighboring positions. Thus, either $v_{1}=v_{3}$ or $v_{2}=v_{4}$. In the first case, pairs $v_{1} v_{2}$ and $v_{2} v_{3}$ contribute to the imprint by exactly one + and one -, and the same is true for the other two pairs, $v_{3} v_{4}$ and $v_{4} v_{1}$. We conclude that the imprint contains exactly two + signs and two - signs. In the second case, the same conclusion holds by an analogous argument.

Now, for $\ell=3$ and for $\ell \geq 5$ we show that $C K(2, \ell)$ are not strongly connected. In particular, we distinguish 4 cases depending on the value of $\ell$ and, for each case, we show two vertices $u$ and $v$ of $C K(2, \ell)$ such that the numbers of + and - signs in the imprints of the two vertices differ (see Table 1 ). Then, by Corollary 2.6, there is no path from $u$ to $v$ in $C K(2, \ell)$. Note that for $\ell=3 r$ with $r \geq 1$, we obtain directed cycles of length 3 without reaching all the vertices, which indicates that the digraph is not strongly connected. For example, for $\ell=3$, see Figure 2 (left).

Lemma 2.8. The diameter of $C K(3,4)$ is $6=2 \ell-2$.

Proof. First, we prove that the diameter is at most 6 . Consider two vertices $u_{1} u_{2} u_{3} u_{4}$ and $v_{1} v_{2} v_{3} v_{4}$. We show that there is either a sequence $y_{1} y_{2}$ or $y_{1}$ such that by concatenating $u_{1} u_{2} u_{3} u_{4}, y_{1} y_{2}$ or $y_{1}$, and $v_{1} v_{2} v_{3} v_{4}$, we obtain a sequence with the property that any contiguous subsequence of length 4 forms a vertex of $C K(3,4)$.

In the first case, $u_{1} u_{2} u_{3} u_{4} y_{1} y_{2} v_{1} v_{2} v_{3} v_{4}$, corresponding to a walk of length 6 , we must have:

(1.1) $y_{1} \neq u_{2}, u_{4}, v_{2}$

(1.2) $y_{2} \neq y_{1}, u_{3}, v_{1}, v_{3}$

(1.3) $v_{1} \neq u_{4}$.

In the second case, $u_{1} u_{2} u_{3} u_{4} y_{1} v_{1} v_{2} v_{3} v_{4}$, corresponding to a walk of length 5 , we must have:

(2.1) $y_{1} \neq u_{2}, u_{4}, v_{1}, v_{3}$;

(2.2) $v_{1} \neq y_{1}, u_{3}$;

(2.3) $v_{2} \neq u_{4}$.

We begin with the case that (1.3) does not hold, that is, $v_{1}=u_{4}$. Then, there is always a possible choice for $y_{1}$ in (2.1), because the alphabet has 4 symbols. Moreover, $v_{1}=u_{4} \neq u_{3}, u_{4}=v_{1} \neq v_{2}$, and (2.2) and (2.3) are satisfied. This assures a walk of length 5 .

Now suppose that $v_{1} \neq u_{4}$. A choice for $y_{1}$ in (1.1) is always possible. Besides, if some of the four symbols $y_{1}, u_{3}, v_{1}, v_{3}$ in (1.2) are equal, a choice for $y_{2}$ is also possible, and then a walk of length 6 is obtained. Otherwise, if $y_{1}, u_{3}, v_{1}, v_{3}$ are different, we consider again the sequence $u_{1} u_{2} u_{3} u_{4} y_{1} v_{1} v_{2} v_{3} v_{4}$, where (2.1) holds because of the choice of $y_{1}$ in (1.1), and so does (2.2). Moreover, we must have $u_{4} \notin\left\{y_{1}, u_{3}\right\}$ (and $v_{1} \neq u_{4}$ ), then $u_{4}=v_{3} \neq v_{2}$ because $y_{1}, u_{3}, v_{1}, v_{3}$ are all different. Thus, (2.3) is fulfilled and we obtain a walk of length 5 .

Finally, it is easy to check that there are vertices at distance 6, for instance, 1012 and 0202.

We proceed with the remaining values of $d$ and $\ell$, and give coincident upper and lower bounds for the diameter of $C K(d, \ell)$.

Lemma 2.9. The diameter of $C K(3, \ell \geq 3)$ with $\ell \neq 4$ is at most $2 \ell-1$.

Proof. We claim that between any two vertices $u$ and $v$ of $C K(3, \ell \geq 3)$ with $\ell \neq 4$, there is always a path of length at most $2 \ell-1$.

In particular, we will show that there is either a sequence $x=x_{1} x_{2} \ldots x_{\ell-1}$ of $\ell-1$ symbols or a sequence $y=y_{1} y_{2} \ldots y_{\ell-2}$ of $\ell-2$ symbols such that by concatenating the sequence of $u$, one of $x$ or $y$, and the sequence of $v$, we obtain a sequence with the property that any contiguous subsequence of length $\ell$ forms a vertex of $C K(3, \ell \geq 3)$ with $\ell \neq 4$.

Given vertices $u$ and $v$, let us first find such a string $x$ of $\ell-1$ symbols. From the definition of a vertex of a cyclic Kautz digraph it follows that, in the desired sequence, the symbol $x_{i}$ must differ from symbols $x_{i-1}, x_{i+1}, u_{i+1}$, and $v_{i}$ (also, $x_{1}$ differs from $u_{\ell}$, and $x_{\ell-1}$ differs from $v_{1}$ ). We adopt the following strategy. We choose the symbols for $x_{1}, x_{2}, \ldots, x_{\ell-1}$ one by one in this order. Our alphabet has 4 symbols, but for each 
$x_{i}$ (with the exception of the last one) there are exactly 3 restrictions given. We therefore can choose the symbols for $x_{1}, x_{2}, \ldots, x_{\ell-2}$ that meet the requirements. However, a problem may arise when choosing the symbol for $x_{\ell-1}$, for which we need to choose a symbol that differs from $u_{\ell}, x_{\ell-2}, v_{1}$, and $v_{\ell-1}$. We distinguish two cases. If any two of these 4 symbols are the same, there remains a symbol which can be assigned to $x_{\ell-1}$ and the constructed sequence $x$ of length $\ell-1$ satisfies the requirements.

Otherwise, if all the 4 symbols $u_{\ell}, x_{\ell-2}, v_{1}$, and $v_{\ell-1}$ differ, say $u_{\ell}=\alpha_{1}, x_{\ell-2}=\alpha_{2}, v_{1}=\alpha_{3}$, and $v_{\ell-1}=\alpha_{4}$, we cannot assign a symbol to $x_{\ell-1}$ that would satisfy the requirements. We observe the following:

(1) $u_{\ell} \neq v_{1}$

(2) $u_{\ell}=\alpha_{1}$ implies $u_{\ell-1} \neq \alpha_{1}$;

(3) $x_{\ell-2}=\alpha_{2}$ implies $u_{\ell-1} \neq \alpha_{2}$.

In this case, instead of searching a sequence $x$ of $\ell-1$ symbols, we find a sequence $y$ of $\ell-2$ symbols as follows. In the desired string, a symbol $y_{i}$ must differ from the symbols $y_{i-1}, y_{i+1}, u_{i+1}$, and $v_{i+1}$ (also, $y_{1}$ differs from $u_{\ell}$, and $y_{\ell-2}$ differs from $\left.v_{1}\right)$. Moreover, since $y$ has length $\ell-2$, the subsequence $u_{\ell} y_{1} \ldots y_{\ell-2} v_{1}$ also needs to be a vertex of $C K(3, \ell \geq 3)$ with $\ell \neq 4$, thus $u_{\ell}$ must differ from $v_{1}$. The condition $u_{\ell} \neq v_{1}$ is satisfied by the above observation (1). To choose the symbols for $y_{1}, y_{2}, \ldots, y_{\ell-2}$ we adopt a similar strategy as above. Again, we can choose the symbols for $y_{1}, y_{2}, \ldots, y_{\ell-3}$ one by one in this order and meet the requirements. Finally, for $y_{\ell-2}$ we need to choose a symbol that differs from $u_{\ell-1}, y_{\ell-3}, v_{1}$, and $v_{\ell-1}$. Since the previous search for $x$ failed, we know that $v_{1}=\alpha_{3}, v_{\ell-1}=\alpha_{4}$, and $u_{\ell-1} \neq \alpha_{1}, \alpha_{2}$. This implies that $u_{\ell-1}$ has the same symbol as either $v_{1}$ or $v_{\ell-1}$, and thus there remains one symbol which can be assigned to $y_{\ell-2}$.

Therefore, we can either find a sequence $x$ of length $\ell-1$, or a sequence $y$ of length $\ell-2$. In both cases this gives us an upper bound of $2 \ell-1$ on the length of the shortest path between any pair of vertices of $C K(3, \ell \geq 3)$ with $\ell \neq 4$.

We use a strategy very similar in the proofs of Lemmas 2.10 and 2.11. In order to avoid repetitions, we omit the proof of the former and only give the one of the latter.

Lemma 2.10. The diameter of $C K(d \geq 3,3)$ is at most $5=2 \ell-1$.

Lemma 2.11. The diameter of $C K(d \geq 4, \ell \geq 4)$ is at most $2 \ell-2$.

Proof. We show that for any pair of vertices $u$ and $v$ of $C K(d \geq 4, \ell \geq 4)$, there is always a path of length at most $2 \ell-2$ or $2 \ell-3$. In particular, we show that there is either a sequence $y=y_{1} y_{2} \ldots y_{\ell-2}$ of $\ell-2$ symbols or a sequence $z=z_{1} z_{2} \ldots z_{\ell-3}$ of $\ell-3$ symbols, such that the concatenation of $u$, one of $y$ or $z$, and $v$ forms a sequence such that any contiguous subsequence of length $\ell$ is a vertex of $C K(d \geq 4, \ell \geq 4)$.

Given vertices $u$ and $v$, we distinguish two cases depending on whether symbols $u_{\ell}$ and $v_{1}$ differ or not. Let us first assume that $u_{\ell} \neq v_{1}$. We construct sequence $y$ of $\ell-2$ symbols one by one, respecting the following restrictions. Symbol $y_{1}$ must differ from symbols $u_{2}, u_{\ell}$, and $v_{2}$. In general, $y_{i}$ must differ from $u_{i+1}, y_{i-1}$, and $v_{i+1}$. Finally, $y_{\ell-2}$ must also differ from $v_{1}$. Since for each of $y_{i}$ we have at most 4 restrictions, and the alphabet contains 5 symbols, we can always find a symbol to assign to $y_{i}$. These restrictions ensure that the constructed sequence $y$ satisfies that each subsequence of $\ell$ symbols of $u, y$ or of $y, v$ forms a vertex of $C K(d \geq 4, \ell \geq 4)$. Moreover, the assumption that $u_{\ell} \neq v_{1}$ ensures that also sequence $u_{\ell} y_{1} \ldots y_{\ell-2} v_{1}$ forms a vertex of $C K(d \geq 4, \ell \geq 4)$.

Let us now assume that $u_{\ell}=v_{1}$. This implies that no sequence $y$ of length $\ell-2$ satisfying the above property can be found, since $u_{\ell} y_{1} \ldots y_{\ell-2} v_{1}$ is not a vertex of $C K(d \geq 4, \ell \geq 4)$. However, this also implies that $u_{\ell}=v_{1} \neq v_{2}$ and that $v_{1}=u_{\ell} \neq u_{\ell-1}$. We construct sequence $z$ of $\ell-3$ symbols one by one, respecting analogous restrictions as in the previous case. Again, due to the fact that the alphabet contains 5 symbols, we can find $z$, such that subsequences of $\ell$ symbols of $u, z$ or of $z, v$ form a vertex of $C K(d \geq 4, \ell \geq 4)$. Besides, since $u_{\ell}=v_{1}$, we observe that $u_{\ell} \neq v_{2}$ and $v_{1} \neq u_{\ell-1}$. Therefore also both sequences $u_{\ell-1} u_{\ell} z_{1} \ldots z_{\ell-3} v_{1}$ and $u_{\ell} z_{1} \ldots z_{\ell-3} v_{1} v_{2}$ form vertices of $C K(d \geq 4, \ell \geq 4)$.

Therefore, we can either find a sequence $y$ of length $\ell-2$, or a sequence $z$ of length $\ell-3$. This gives us an upper bound of $2 \ell-2$ on the diameter of $C K(d \geq 4, \ell \geq 4)$. 
Lemma 2.12. The diameter of $C K(d \geq 3,3)$ is at least $5=2 \ell-1$.

Proof. Let $u=012$ and $v=210$ be two vertices of $C K(d \geq 3,3)$. We argue that $u$ and $v$ are at distance at least 5 as follows. Since the only symbol 2 in $u$ is at position $u_{3}$, but $v_{1}=2$, at least two steps are needed to reach $v$ from $u$. Since $u_{2}=v_{2}$, then $u_{2} u_{3} v_{2}\left(=u_{2} v_{1} v_{2}\right)$ is not a vertex and $v$ cannot be reached from $u$ in two steps. Also, since $u_{3}=v_{1}$ and $u_{3} v_{1} v_{2}$ is not a vertex, thus also three steps are not enough to reach $v$ from $u$. Finally, since $u_{3}=v_{1}$ and $u_{3} x v_{1}$ is not a vertex for any symbol $x$, thus $v$ cannot be reached from $u$ in four steps.

Lemma 2.13. The diameter of $C K(3, \ell \geq 3)$ with $\ell \neq 4$ is at least $2 \ell-1$.

Proof. For $\ell=3$, it is easy to verify that the vertices 012 and 210 of $C K(3,3)$ are at distance 5 . Let then $\ell \geq 5$. We distinguish two cases depending on the parity of $\ell$, and in both we describe a pair of vertices $u$ and $v$ of $C K(3, \ell \geq 5)$ that are at distance at least $2 \ell-1$.

First, let $\ell$ be odd. Consider $u=0101 \ldots 012$, and $v=210 \ldots 1010$ of $C K(3, \ell \geq 5)$, where we underlined the symbols that are repeated. Since the only symbol 2 in $u$ is at position $u_{\ell}$, but $v$ contains 2 at position $v_{1}$, at least $\ell-1$ steps are needed to reach $v$ from $u$. However, $\ell-1$ steps are also not enough, since $u_{\ell-1}=1=v_{\ell-1}$, and thus sequence $u_{\ell-1} u_{\ell} v_{2} \ldots v_{\ell-1}\left(=u_{\ell-1} v_{1} v_{2} \ldots v_{\ell-1}\right)$ is not a vertex of $C K(3, \ell \geq 5)$. For the sake of contradiction, assume that there is a path of length $\ell+z$, for some $0 \leq z<\ell-1$. Then, there must exist a sequence of $z$ symbols $x=\left(x_{1}, x_{2}, \ldots, x_{z}\right)$, such that any contiguous subsequence of length $\ell$ of $u, x, v$ forms a vertex of $C K(3, \ell \geq 5)$. For $z=\ell-2$ no such sequence $x$ exists, since $u_{\ell}=2=v_{1}$, which implies that $u_{\ell} x_{1} \ldots x_{z} v_{1}$ is not a vertex of $C K(3, \ell \geq 5)$. If $z$ is odd, there is no such sequence $x$, since $u_{\ell-1}=1=v_{\ell-2-z}$, which implies that $u_{\ell-1} u_{\ell} x_{1} \ldots x_{z} v_{1} \ldots v_{\ell-2-z}$ is not a vertex of $C K(3, \ell \geq 5)$. If $z$ is even, assume for the sake of contradiction that there is such a sequence $x$. First observe that $x$ cannot contain any symbol 0 or 1 as follows. Since any subsequence of $u, x, v$ of $\ell$ consecutive symbols must form a vertex of $C K(3, \ell \geq 5)$, the symbol at position $x_{i}$ must differ from the symbols at positions $u_{i+1}$ and $v_{\ell-1-z+i}$. Thus, at position $x_{i}$, cannot be 0: If $i$ is odd, also $\ell-1-z+i$ is odd and thus $v_{\ell-1-z+i}=0$; otherwise if $i$ is even, then $i+1$ is odd and thus $u_{i+1}=0$. Note that $0 \leq z<\ell-1$ implies $\ell-1-z+i>1$, and $i \leq z$, thus also $i+1 \leq \ell-1$. Similarly, the symbol at position $x_{i}$ cannot be 1 : If $i$ is odd, $i+1$ is even and $u_{i+1}=1$; otherwise, if $i$ is even, $\ell-1-z+i$ is even and $v_{\ell-1-z+i}=1$. Therefore, since the size of the alphabet is 4 , sequence $x$ consists only of 2's and 3's. Since $u_{\ell}=2=v_{1}$, both $x_{1}$ and $x_{z}$ must be 3. Then, $x_{2}=x_{z-1}=2$, and $x_{3}=x_{z-2}=3$, and so on. Since $z$ is even, sequence $x$ has even length, and thus $x_{z / 2}=x_{z / 2+1}$ (the same symbols meet in the middle of $x$ ), which is not possible and we get a contradiction.

Now, let $\ell$ be even and let us proceed similarly. Consider $u=10 \underline{20} \underline{20} \ldots \underline{2012}$, and $v=213 \underline{02} \underline{02} \ldots \underline{02010}$ of $C K(3, \ell \geq 5)$, where the underlined symbols mean that they are repeated. (For example, for $\ell=6$, $u=102012$, and $v=213010$.)

Since vertex $v$ starts with symbols 21 , but vertex $u$ does not contain this pattern, at least $\ell-1$ steps are needed to reach $v$ from $u$. However, $\ell-1$ steps are also not enough, since $u_{\ell-1}=1=v_{\ell-1}$, and thus sequence $u_{\ell-1} u_{\ell} v_{2} \ldots v_{\ell-1}\left(=u_{\ell-1} v_{1} \ldots v_{\ell-1}\right)$ is not a vertex of $C K(3, \ell \geq 5)$. Also $\ell$ steps do not suffice, since $u_{\ell}=2=v_{1}$, and thus $u_{\ell} v_{1} \ldots v_{\ell-1}$ is not a vertex of $C K(3, \ell \geq 5)$. Again, assume for the sake of contradiction that there is a path of length $\ell+z$ connecting $u$ and $v$, for some $1 \leq z<\ell-1$. Then, there must exist a sequence of $z$ symbols $x=\left(x_{1}, x_{2}, \ldots, x_{z}\right)$ such that any contiguous subsequence of length $\ell$ of $u, x, v$ forms a vertex of $C K(3, \ell \geq 5)$. We distinguish two cases depending on the parity of $z$. If $z$ is odd, we distinguish three cases depending on $z$ (the length of $x$ ), and for each we argue why there cannot be such a sequence $x$.

- $z<\ell-5$ : Independent of $x$, subsequence $u_{\ell-2} u_{\ell-1} u_{\ell} x_{1} \ldots x_{z} v_{1} \ldots v_{\ell-3-z}$ is not a vertex of $C K(3, \ell \geq 5)$, since $u_{\ell-2}=0=v_{\ell-3-z}$.

- $z=\ell-5$ : Independent of $x$, subsequence $u_{\ell-3} \ldots u_{\ell} x_{1} \ldots x_{z} v_{1}$ is not a vertex of $C K(3, \ell \geq 5)$, since $u_{\ell-3}=2=v_{1}$.

- $z=\ell-3$ : We observe that there are the following restrictions on the symbols in $x$ : Since for each $i$, both $u_{i+1} \ldots u_{\ell} x_{1} \ldots x_{i}$ and $x_{i} \ldots x_{z} v_{1} \ldots v_{i+2}$ must be vertices of $C K(3, \ell \geq 5)$, symbol $x_{i}$ must differ from both $u_{i+1}$ and $v_{i+2}$. Moreover, $x_{1}$ must differ from $u_{\ell}$, and $x_{z}$ must differ from $v_{1}$. Considering $u$ and 
$v$, this implies that $x$ can only consist of symbols 1 and 3 , with $x_{1}=1$ and $x_{z}=3$. However, since the two symbols in $x$ must alternate, and $z$ is odd, all these conditions cannot be satisfied and we get a contradiction.

If $z$ is even, we distinguish three cases depending on $z$ (the length of $x$ ), and for each we identify a subsequence of $u, x, v$ of length $\ell$ that does not form a vertex of $C K(3, \ell \geq 5)$ :

- $z<\ell-4: u_{\ell} x_{1} \ldots x_{z} v_{1} \ldots v_{\ell-1-z}$ is not a vertex, since $u_{\ell}=2=v_{\ell-1-z}$.

- $z=\ell-4: u_{\ell-1} u_{\ell} x_{1} \ldots x_{z} v_{1} v_{2}$ is not a vertex, since $u_{\ell-1}=1=v_{2}$.

- $z=\ell-2: u_{\ell} x_{1} \ldots x_{z} v_{1}$ is not a vertex, since $u_{\ell}=2=v_{1}$.

Therefore, in both cases (odd and even $\ell$ ), we identified a pair of vertices of $C K(3, \ell \geq 3)$ with $\ell \neq 4$ that are at distance at least $2 \ell-1$, thus bounding the diameter of $C K(3, \ell \geq 3)$ with $\ell \neq 4$ from below.

\begin{tabular}{|c|c|c|c|c|c|}
\hline$d \quad \ell$ & 1 & 2 & 3 & 4 & $\geq 5$ \\
\hline 1 & & 1 & $\nexists$ & 1 & \\
\hline 2 & & \multirow{3}{*}{$\ell$} & $\infty$ & $2 \ell-1$ & $\infty$ \\
\hline 3 & & & \multirow{2}{*}{$2 \ell-1$} & \multirow{2}{*}{$2 \ell-2$} & $2 \ell-1$ \\
\hline$\geq 4$ & & & & & \\
\hline
\end{tabular}

Figure 4: Summary of the diameter of $C K(d, \ell)$, depending on the values of $d$ and $\ell$.

Lemma 2.14. The diameter of $C K(d \geq 4, \ell \geq 4)$ is at least $2 \ell-2$.

Proof. Consider $u=\ldots 0101012$ ( $u$ begins with 01 if $\ell$ is odd and with 10 if $\ell$ is even), and $v=1320202 \ldots$ of $C K(d \geq 4, \ell \geq 4)$. We show that the shortest path from $u$ to $v$ in $C K(d \geq 4, \ell \geq 4)$ has length at least $2 \ell-2$ as follows. Since vertex $v$ starts with symbols 13 , but vertex $u$ does not contain this pattern, at least $\ell-1$ steps are needed to reach $v$ from $u$. Also, since $u_{\ell} \neq v_{1}, \ell-1$ steps are not enough. For the sake of contradiction, assume that there is a path of length $\ell+z$, for some $0 \leq z<\ell-2$. Then, there must exist a sequence of $z$ symbols $x=\left(x_{1}, x_{2}, \ldots, x_{z}\right)$ such that any contiguous subsequence of length $\ell$ of $u, x, v$ forms a vertex of $C K(d \geq 4, \ell \geq 4)$. We distinguish four cases depending on the parity of $z$ and $\ell$, and for each we identify a subsequence of $u, x, v$ of length $\ell$ that does not form a vertex of $C K(d \geq 4, \ell \geq 4)$ :

- $z$ odd, $\ell$ odd: $u_{\ell} x_{1} \ldots x_{z} v_{1} \ldots v_{\ell-1-z}$ is not a vertex, since $u_{\ell}=2=v_{\ell-1-z}$.

- $z$ odd, $\ell$ even: $u_{z+2} \ldots u_{\ell} x_{1} \ldots x_{z} v_{1}$ is not a vertex, since $u_{z+2}=1=v_{1}$.

- $z$ even, $\ell$ odd: $u_{z+2} \ldots u_{\ell} x_{1} \ldots x_{z} v_{1}$ is not a vertex, since $u_{z+2}=1=v_{1}$.

- $z$ even, $\ell$ even: $u_{\ell} x_{1} \ldots x_{z} v_{1} \ldots v_{\ell-1-z}$ is not a vertex, since $u_{\ell}=2=v_{\ell-1-z}$.

Therefore, the shortest path from $u$ to $v$ has length at least $2 \ell-2$.

Results 2.3-2.14 determine the diameter of $C K(d, \ell)$ for all the values of $d$ and $\ell$; we summarize them into the following theorem (see Figure 4 for a scheme).

Theorem 2.15. The diameter of $C K(d, \ell)$ is

$$
D(C K(d, \ell))=\left\{\begin{array}{cl}
1 & \text { for } d=1, \ell \geq 2 \text { if } \ell \text { is even; } \\
\ell & \text { for } \ell=1 ; \text { for } d \geq 2, \ell=2 ; \\
2 \ell-1 & \text { for } d=2, \ell=4 ; \text { for } d \geq 3, \ell=3 ; \text { for } d=3, \ell \geq 5 ; \\
2 \ell-2 & \text { for } d=3, \ell=4 ; \text { for } d \geq 4, \ell \geq 4 .
\end{array}\right.
$$

Recall that, for $d=1$ and $\ell \geq 2$ when $\ell$ is odd, the cyclic Kautz digraphs do not exist. Moreover, for $d=2$ and $\ell \geq 3$ when $\ell \neq 4$, the cyclic Kautz digraphs are not strongly connected. 


\section{Conclusions}

In this paper we give the main parameters of a new network called cyclic Kautz digraph $C K(d, \ell)$, which is related to cyclic codes. These parameters are the number of vertices, the number of arcs and the diameter for all the values of $d$ and $\ell$. As cyclic Kautz digraphs $C K(d, \ell)$ are subdigraphs of the well-known Kautz digraphs $K(d, \ell)$, the former inherits some properties of the latter. It would be interesting to explore further properties of cyclic Kautz digraphs, as well as their relations to coding theory.

\section{Acknowledgments.}

We want to thank the anonymous referees for their reviews, which significantly contributed to improve the quality of this publication.

\section{References}

[1] K. Böhmová, C. Dalfó, C. Huemer, The diameter of cyclic Kautz digraphs, Electronic Notes on Discrete Mathematics 49 (2015) 323-330.

[2] A. E. Brouwer, W. H. Haemers, Spectra of Graphs, Springer, New York, 2012.

[3] N. G. de Bruijn, A combinatorial problem, Koninklijke Nederlandse Akademie van Wetenschappen Proc. A49 (1946) $758-764$

[4] J. Fàbrega, M. A. Fiol, Maximally connected digraphs, Journal of Graph Theory 13 (1989) 657-668

[5] M. A. Fiol, J. L. A. Yebra, I. Alegre, Line digraph iterations and the $(d, k)$ digraph problem, IEEE Transactions on Computers C-33 (1984) 400-403.

[6] W. H. Kautz, Bounds on directed $(d, k)$ graphs, in Theory of Cellular Logic Networks and Machines, AFCRL-68-0668 Final Rep. (1968) 20-28.

[7] W. H. Kautz, Design of optimal interconnection networks for multiprocessors, in Architecture and Design of Digital Computers, Nato Advanced Summer Institute (1969) 249-272.

[8] J. H. van Lint, An Introduction to Coding Theory, 3rd edition, Springer-Verlag, New York, 1999.

[9] N. J. A. Sloane, The On-Line Encyclopedia of Integer Sequences, http://oeis . org. 